\title{
SÍNTESE E CARACTERIZAÇÃO ESTRUTURAL DE PIGMENTOS CERÂMICOS A BASE DE CROMITA POR MÉTODO QUÍMICO
}

\author{
J. H. G. Rangel' ${ }^{1}$ E. B. S. Pereira ${ }^{2}$, P. R. G. Gonçalves Júnior ${ }^{3}$, M. M. Oliveira ${ }^{4}$, J. S. Vasconcelos ${ }^{5}$ e E. Longo ${ }^{6}$ \\ 1,2,3,4,5 Instituto Federal do Maranhão (IFMA), ${ }^{6}$ Instituto de Química - Universidade Estadual Paulista (UNESP) \\ E-mail: hiltonrangel@ifma.edu.br ${ }^{1}$, edilsonbp@gmail.com ${ }^{2}$, paulogoncalvesjr@ifma.edu.br ${ }^{3}$, \\ marcelo@ifma.edu.br ${ }^{4}$,jomar@ifma.edu.br ${ }^{5}$ e elson@iq.unesp.br ${ }^{6}$
}

Artigo submetido em julho/2012 e aceito em agosto/2012

\section{RESUMO}

Os pigmentos cerâmicos são substâncias inorgânicas coloridas que durante o processo de dispersas nos esmaltes cerâmicos e posterior calcinação, se mantêm estáveis frente ao ataque físico e químico. Por conseguinte, são compostos cristalinos aplicados na indústria cerâmica para colorir bases vítreas. Neste trabalho foi utilizado o método Pechini na obtenção do pigmento $\mathrm{CuCr} 2 \mathrm{O} 4$ com tratamento térmico nas temperaturas de 800,900 e $1000^{\circ} \mathrm{C}$. Os pós pigmentantes foram caracterizados nos aspectos estruturais, morfológicos e colorimétricos. A análise térmica realizada no precursor amorfo em um TG/DTA revela a perda de massa em todo o intervalo de temperatura investigado, com pico exotérmico característico da eliminação da composição orgânica do precursor em torno de $300^{\circ} \mathrm{C}$. A evolu- ção das fases cristalinas foi investigada por DRX, utilizando um difratometro com radiação $\mathrm{k}$ ? do Cu e monocromador de grafite onde se observou a presença de fases cristalinas correspondentes ao $\mathrm{Cr} 2 \mathrm{O} 3$ e $\mathrm{CuCr} 2 \mathrm{O} 4$. As medidas de área superficial específica dos pós pigmentantes foram realizadas em um equipamento Micromeritcs modelo ASAP 2000, utilizando N2 como gás de adsorção/desorção. As medidas colorimétricas dos pigmentos foram realizadas em um colorímetro Gretac Macbeth Color-eye spectrophotometer 2180/ 2180UV, nos padrões CIELAB. Com base nos resultados obtidos verifica-se a estabilidade térmica dos pós pigmentantes de coloração verde, o que o habilita como alternativa aos materiais atualmente empregados na indústria de pisos cerâmicos.

PALAVRAS-CHAVE: Espinélios, Cromita de cobre, Pigmento inorgânico, Pechini.

\section{SYNTHESIS AND STRUCTURAL CHARACTERIZATION OF CHROMITES CERAMIC PIGMENTS BY CHEMICAL METHOD}

\begin{abstract}
The ceramic pigments are colored inorganic substances that during the process of dispersion in the ceramic glazes and subsequent calcination, are stable against physical and chemical attack. Therefore, they are crystalline compounds applied in the ceramic industry for coloring vitreous base. In this study, the Pechini method was used for obtaining the pigment $\mathrm{CuCr} 2 \mathrm{O} 4$ with heat treatment at the temperatures of 800,900 and $1000^{\circ} \mathrm{C}$. The powder pigments were characterized on their structural, morphological and colorimetric aspects. The thermal analysis conducted on an amorphous precursor in a TG / DTA indicates the weight loss in the entire temperature range investigated, with characteristic exothermic peak of the elimination of the organic composition of the precursor around $300^{\circ} \mathrm{C}$. The
\end{abstract}

development of the crystalline phases were investigated by $X R D$, using a diffractometer with Cu Ka radiation and graphite monochromator, where it was observed the presence of crystalline phases corresponding to $\mathrm{Cr} 2 \mathrm{O} 3$ and CuCr204. The measurements of the specific surface area of the powders pigments were carried out in an equipment Micromeritcs, model ASAP 2000, using N2 as gas of adsorption/desorption. The colorimetric measurements of the pigments were made in a colorimeter Gretac Macbeth Color-eye spectrophotometer 2180 / 2180 UV in CIELAB standards. Based on the obtained results, it can be verified the thermal stability of the powder pigments of green coloration, which enables it as an alternative to the materials currently used in the manufacture of ceramic tiles.

KEY-WORDS: Spinels, Chromite Copper, Inorganic Pigment, Pechini. 


\section{SÍNTESE E CARACTERIZAÇÃO ESTRUTURAL DE PIGMENTOS CERÂMICOS A BASE DE CROMITA POR MÉTODO QUÍMICO}

\section{INTRODUÇÃO}

Os pigmentos cerâmicos são definidos como particulado sólido de natureza inorgânico, com cores variadas, que se mantêm insolúvel no substrato no qual é depositado e que não reaja quimicamente ou fisicamente (LIEC, 2003).

São divididos em dois grupos: Pigmentos orgânicos ou naturais e inorgânicos ou sintéticos. Ambos os grupos têm sua grande e crescente importância econômica, contudo, as propriedades gerais, são muito diferentes entre os grupos. Os orgânicos têm uma vasta gama de tons muito brilhante e um poder de coloração extraordinário, usado em larga escala em trabalhos estéticos e apresentando baixo custo. Contudo, com um inconveniente para a utilização deste tipo de pigmento em produção seriada é a reprodutibilidade, pois apresenta características pouco homogênea e diversos tipos de quantidades de impurezas (BANDIOLE, 1998).

Os pigmentos inorgânicos são os mais utilizado industrialmente, podem ser produzidos com um elevado grau de pureza química e uniformidade, apresentam maior estabilidade térmica e química, o que permite a coloração de materiais obtidos à elevada temperatura, e são mais caros que os pigmentos naturais. A propriedade mais importante a ser considerada é a capacidade de desenvolver cor (BANDIOLE, 1998).

Devido a condicionantes como os sócio-econômicos, sanitários, meio-ambiente e à necessidade de se aumentar o poder pigmentante das estruturas atualmente conhecidas, a investigação no campo dos pigmentos cerâmicos tem amplo campo e a rota de síntese de pigmentos nanométricos é uma das grandes áreas de pesquisa.

A cromita de cobre $\mathrm{CuCr}_{2} \mathrm{O}_{4}$ apresenta estrutura cristalina do tipo espinélio, onde os átomos de $\mathrm{Cu}^{2+}$ ocupam os sítios tetraédricos e os átomos de $\mathrm{Cr}^{3+}$ ocupam os sítios octaédricos, e pode ser obtida usando a razão estequiométrica $\mathrm{M}^{2+} / \mathrm{M}^{3+}=0,5$ (CUI et al., 2005).

Dollase e O'Neill (1997) avaliaram a formação do $\mathrm{CuCr}_{2} \mathrm{O}_{4}$, a partir da mistura de $\mathrm{Cr}_{2} \mathrm{O}_{3}$ e CuO, utilizando o refinamento Rietveld e relatam a presença de $6 \%$ de $\mathrm{CuCrO}_{2}, 2 \%$ de $\mathrm{Cr}_{2} \mathrm{O}_{3}$ e $92 \%$ de $\mathrm{CuCr}_{2} \mathrm{O}_{4}$.

$\mathrm{O} C r^{3+}$, quando em sítio octaédrico, apresenta quatro transições possíveis na região do visível. A primeira transição ocorre em cerca de $570 \mathrm{~nm}$ e é caracterizada como ${ }^{4} \mathrm{~A}_{2 \mathrm{~g}}\left({ }^{4} \mathrm{~F}\right) \rightarrow{ }^{4} \mathrm{~T}_{2 \mathrm{~g}}\left({ }^{4} \mathrm{~F}\right)$, a segunda ocorre próximo de $440 \mathrm{~nm}$ e é caracterizada como ${ }^{4} \mathrm{~A}_{2 \mathrm{~g}}\left({ }^{4} \mathrm{~F}\right) \rightarrow{ }^{4} \mathrm{~T}_{1 \mathrm{~g}}\left({ }^{4} \mathrm{~F}\right)$ e as duas últimas ocorrem em torno de $640-740 \mathrm{~nm}$ e são caracterizadas como ${ }^{4} \mathrm{~A}_{2 \mathrm{~g}}\left({ }^{4} \mathrm{~F}\right) \rightarrow{ }^{4} \mathrm{~T}_{1 \mathrm{~g}}\left({ }^{2} \mathrm{G}\right)$ e ${ }^{4} \mathrm{~A}_{2 \mathrm{~g}}\left({ }^{4} \mathrm{~F}\right) \rightarrow{ }^{2} \mathrm{E}\left({ }^{2} \mathrm{G}\right)$ e classificadas como transições de spin proibido (DONDI et al., 2006; SILVA, 2008).

\section{MATERIAIS E MÉTODOS}

Utilizou-se o método dos precursores poliméricos na preparação da solução precursora do óxido $\mathrm{CuCr}_{2} \mathrm{O}_{4}$, consistindo na quelação entre os cátions metálicos e o ácido cítrico e a poliesterificação do ácido hidroxicarboxílico com etilenoglicol $\left(\mathrm{C}_{2} \mathrm{H}_{6} \mathrm{O}_{3}\right)$ em uma solução ligeiramente acidificada, A Tabela 1 descreve os reagentes utilizados no preparo do Precursor polimérico. 
Tabela 1. Reagentes utilizados para obtenção do pigmento.

\begin{tabular}{lll}
\hline PRODUTO & FÓRMULA & PUREZA \\
\hline Ácido Cítrico & $\mathrm{C}_{6} \mathrm{H}_{8} \mathrm{O}_{7} \cdot \mathrm{H}_{2} \mathrm{O}$ & $99,5 \%$ P.A \\
Carbonato de Cobre & $\mathrm{CuCO}_{3} \cdot \mathrm{Cu}(\mathrm{OH})_{2} \mathrm{nH}_{2} \mathrm{O}$ & P.A \\
Ácido Nítrico & $\mathrm{HNO}_{3}$ & P.A \\
Hidróxido de Crômio & $\left(\mathrm{CH}_{3} \mathrm{CO}_{2}\right)_{7} \mathrm{Cr}_{3}(\mathrm{OH})_{2}$ & P.A \\
Etilenoglicol & $\mathrm{C}_{2} \mathrm{H}_{6} \mathrm{O}_{3}$ & $99,5 \%$ P.A \\
Água Destilada & $\mathrm{H}_{2} \mathrm{O}$ & P.A \\
\hline
\end{tabular}

\section{Preparação do Citrato de Cobre}

O citrato de cobre foi selecionado para ser o precursor catiônico na preparação das resinas precursoras para obtenção do $\mathrm{CuCr}_{2} \mathrm{O}_{4}$. O citrato de cobre utilizado foi preparado de acordo com procedimento a seguir: dissolveu-se aproximadamente $0,167 \mathrm{~mol}$ de ácido cítrico em $500 \mathrm{~mL}$ de água destilada, em temperatura ambiente $\left(30^{\circ} \mathrm{C}\right)$. Em seguida, solubilizou-se $0,017 \mathrm{~mol}$ de carbonato de cobre em $50 \mathrm{~mL}$ de ácido nítrico concentrado. Juntou-se as soluções em um recipiente de $500 \mathrm{~mL}$ e completou-se o volume e levouse para aquecer sob agitação constante à temperatura de $50^{\circ} \mathrm{C}$.

Foi feita a gravimetria do citrato de cobre para se obter a relação exata da quantidade em gramas, de $\mathrm{CuO}$ por $\mathrm{mL}$ de citrato.

As gravimetrias foram feitas, em atmosfera ambiente, utilizando cadinhos de alumina à temperatura de $800^{\circ} \mathrm{C}$ por duas horas. Verificado a concentração de $\mathrm{Cu}$ na solução por meio da gravimetria adicionou-se o $\left(\mathrm{CH}_{3} \mathrm{CO}_{2}\right)_{7} \mathrm{Cr}_{3}(\mathrm{OH})_{2}$ na proporção de 2 mols de $\mathrm{Cr}$ para $1 \mathrm{~mol}$ de $\mathrm{Cu}$.

\section{Preparação da Resina Precursora}

A solução foi preparada de acordo com o fluxograma abaixo, sintetizado com base do método dos precursores poliméricos, utilizando os reagentes descritos na Tabela 1. Utilizou-se uma razão molar (Ácido cítrico:Metal) de 3:I e a quantidade em massa de ácido cítrico:etilenoglicol de 60:40 respectivamente.

Após a calcinação da resina a 350 $\mathrm{C}$ durante duas horas, no forno mufla, com taxas de aquecimento de 3 ㄷ/min, para obtenção do precursor amorfo, fez-se a maceração do mesmo, em almofariz de porcelana. Os pós obtidos foram tratados termicamente a diferentes temperaturas $(800,900$ e 1000 으 $)$ por duas horas com taxa de aquecimento a $10^{\circ} \mathrm{C} / \mathrm{min}$.

\section{Caracterização dos pós de $\mathrm{CuCr}_{2} \mathrm{O}_{4}$}

A analise termogravimétrica do precursor amorfo foi realizada utilizando um DTA, NETZSCH STA 409C, com razão de aquecimento de $10{ }^{\circ} \mathrm{C} / \mathrm{min}$ e intervalo de temperatura da temperatura ambiente a $1050^{\circ} \mathrm{C}$ em atmosfera oxidadnte.

Os pós calcinados foram caracterizados por difração de Raios X - DRX (modelo D - 5000, Siemens) usando a radiação k $\alpha$ do $\mathrm{Cu}$ e monocromador de grafite. Na determinação das características microestruturais do pigmento foi utilizado um microscópio eletrônico de varredura, ZEISS DSM - modelo 940 A.

As medidas de área superficial específica dos pós pigmentantes foram realizadas em um equipamento Micromeritcs modelo ASAP 2000, utilizando $N_{2}$ como gás de adsorção/desorção. 
As medidas colorimétricas dos pigmentos foram realizadas em um colorímetro Gretac Macbeth Color-eye spectrophotometer 2180/2180UV, nos padrões CIELAB (utilizando iluminação D65).

O teste da estabilidade do pigmento obtido foi realizado com a aplicação de $3 \%$ em peso de pigmento para $97 \%$ em peso de esmalte industrial. A mistura foi homogeneizada e tratada termicamente segundo o roteiro: $500^{\circ} \mathrm{C}$ com taxa de aquecimento de $10^{\circ} \mathrm{C} / \mathrm{min}$, em seguida $1180^{\circ} \mathrm{C} / 1 \mathrm{~h}$ a $15^{\circ} \mathrm{C} / \mathrm{min}$ e resfriado a $10^{\circ} \mathrm{C} / \mathrm{min}$ a temperatura ambiente.

\section{RESULTADOS E DISCUSSÃO}

\section{Difração de Raios X}

Os resultados de DRX indicam a formação de fase cristalina à baixa temperatura, com a formação da fase eskolaita do $\mathrm{Cr}_{2} \mathrm{O}_{3}$ (JCPDS\#38-1479) e tenorita do CuO (JCPDS \#45-0937) a 350ㄷ. O difratograma dos pós calcinados a 800,900 e $1000^{\circ} \mathrm{C}$ durante duas horas apresentado na Figura 1 demonstra a presença majoritária da fase espinélio da cromita com a elevação da temperatura de calcinação, porém com a permanência da presença da fase cristalina da eskoleita.

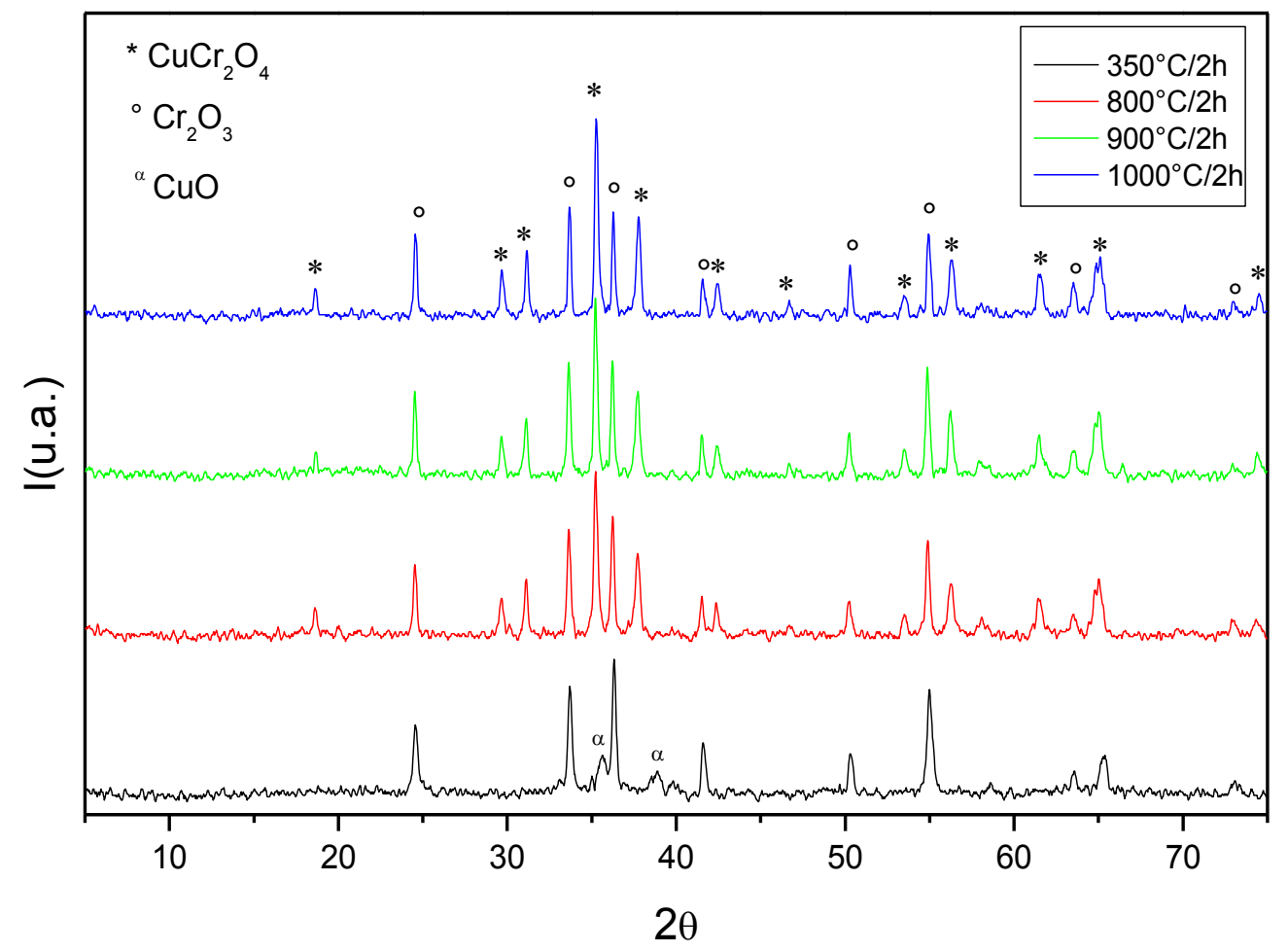

Figura 1. Difratograma dos pós obtidos após calcinação em diferentes temperaturas.

A elevação da temperatura de calcinação dos pós promoveu um aumento no grau de cristalização da fase espinélio da cromita de cobre observado pelo cálculo da largura à meia altura (FWHM) do pico de maior intensidade da fase utilizando o software Origin 8.0, correspondente ao plano (211) apresentado na Figura 2. 

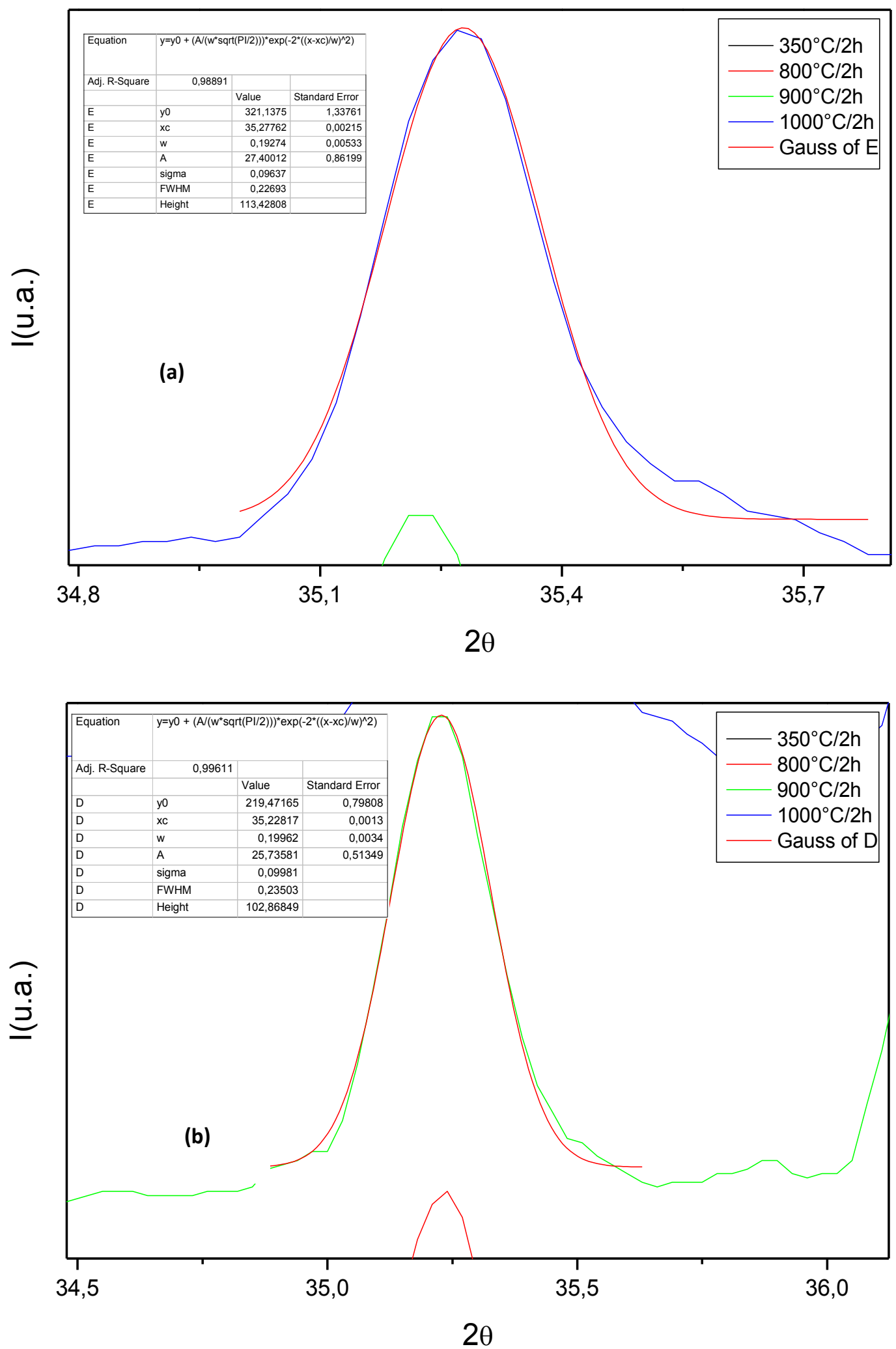


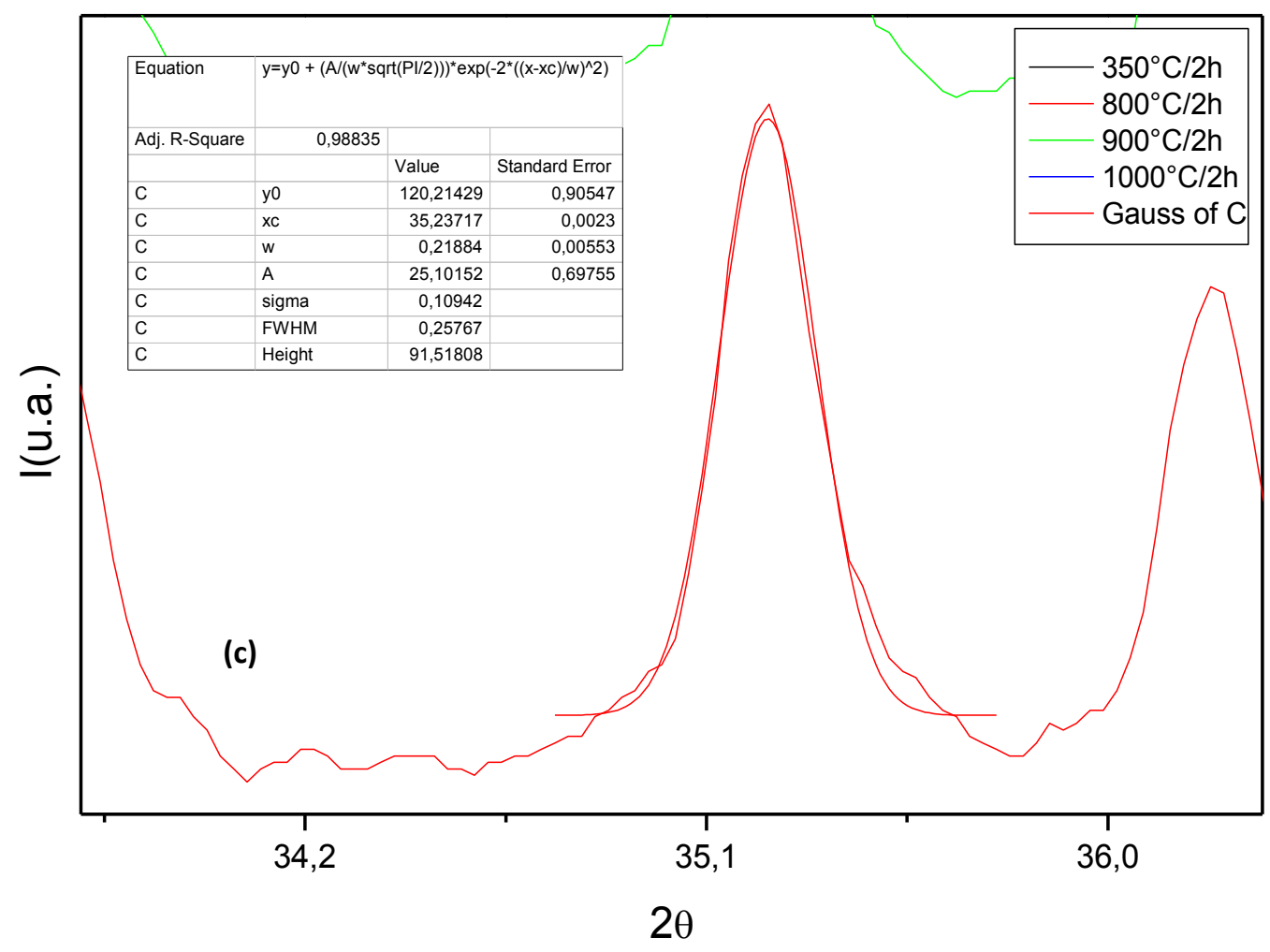

Figura 2. Deconvolução do pico (211) do $\mathrm{CuCr}_{2} \mathrm{O}_{4}$ calcinados nas temperaturas (a) $1000^{\circ} \mathrm{C}$; (b) $900{ }^{\circ} \mathrm{Ce}$ (c) $800^{\circ} \mathrm{C}$.

A coexistência das duas fases cristalinas presentes no pigmento obtido, $\mathrm{Cr}_{2} \mathrm{O}_{3}$ e $\mathrm{CuCr}_{2} \mathrm{O}_{4}$ tetragonal (JCPDS\#34-0424), possibilitam a estabilização da cor verde após aplicação com vidrado nos testes realizados o torna viável ao uso como alternativa aos pigmentos instáveis utilizados na industria cerâmica.

\section{Microscopia Eletrônica de Varredura}

A Microscopia Eletrônica de Varredura (MEV), com aumento de 30.000x e utilizando elétrons secundários, exibida na Figura 3, demonstra que o aumento na temperatura de tratamento dos pós pigmentantes de 800 a $1000^{\circ} \mathrm{C}$ provoca o aumento no tamanho das partículas devido à formação de agregados, 0 que acarreta a diminuição da área superficial. Pode ser observado também a presença de partículas uniformes, arredondadas e aglomeradas e o crescimento do tamanho médio das partículas com a elevação da temperatura de calcinação. 

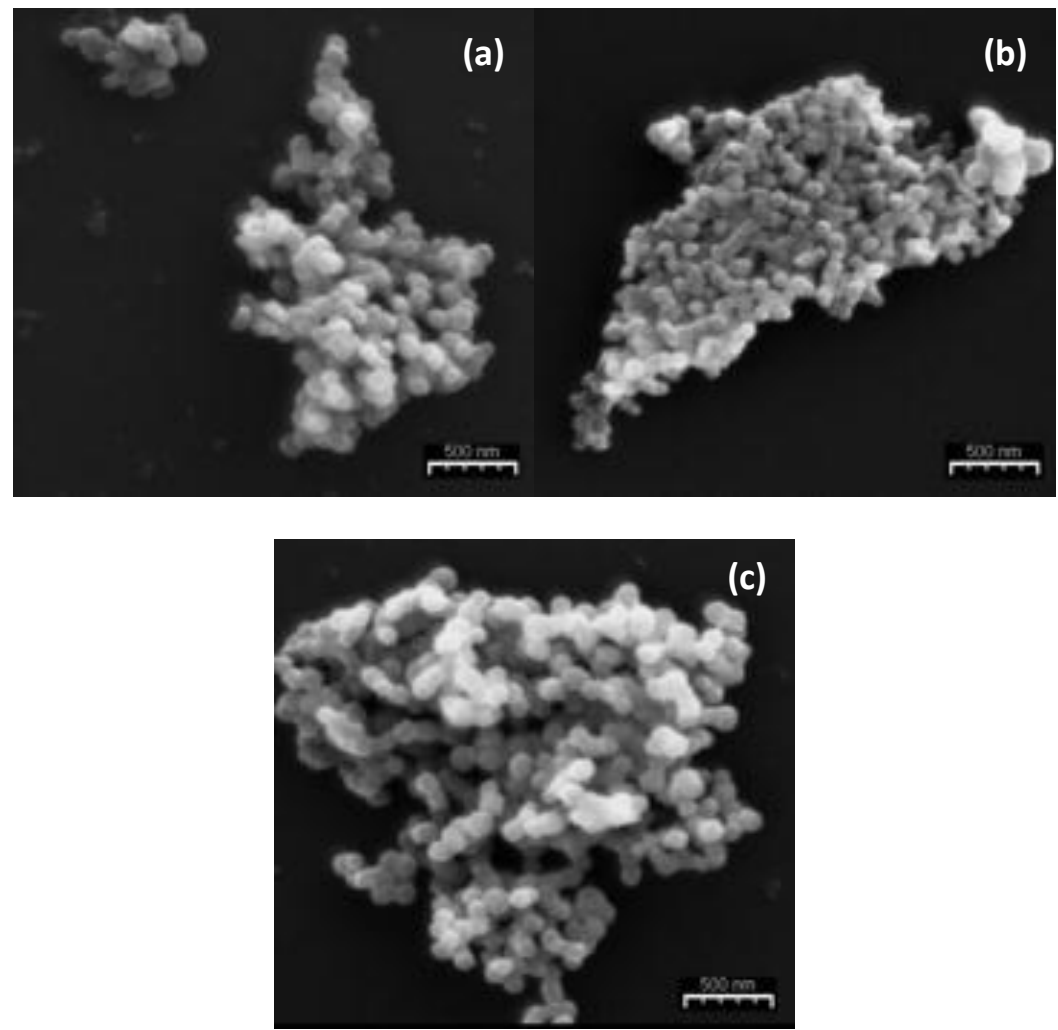

Figura 3. Micrografias dos precursores calcinados a (a) $800^{\circ} \mathrm{C} / 2 \mathrm{~h}$; (b) $900^{\circ} \mathrm{C} / 2 \mathrm{~h}$; (c) $1000^{\circ} \mathrm{C} / 2 \mathrm{~h}$.

\section{Isotermas de adsorção-dessorção}

A análise morfológica por adsorção/dessorção de $N_{2}$ dos pós obtidos, mostrada na Figura 4 , indicam que o material calcinado a $1000^{\circ} \mathrm{C}$ por $2 \mathrm{~h}$ apresenta isoterma do tipo III, característica de sistemas com área superficial inferior a $5 \mathrm{~m}^{2} / \mathrm{g}$ e histerese $\mathrm{H} 1$, correspondente a presença de poros em forma de cilindros abertos e cuja presença indica a existência de aglomerados.

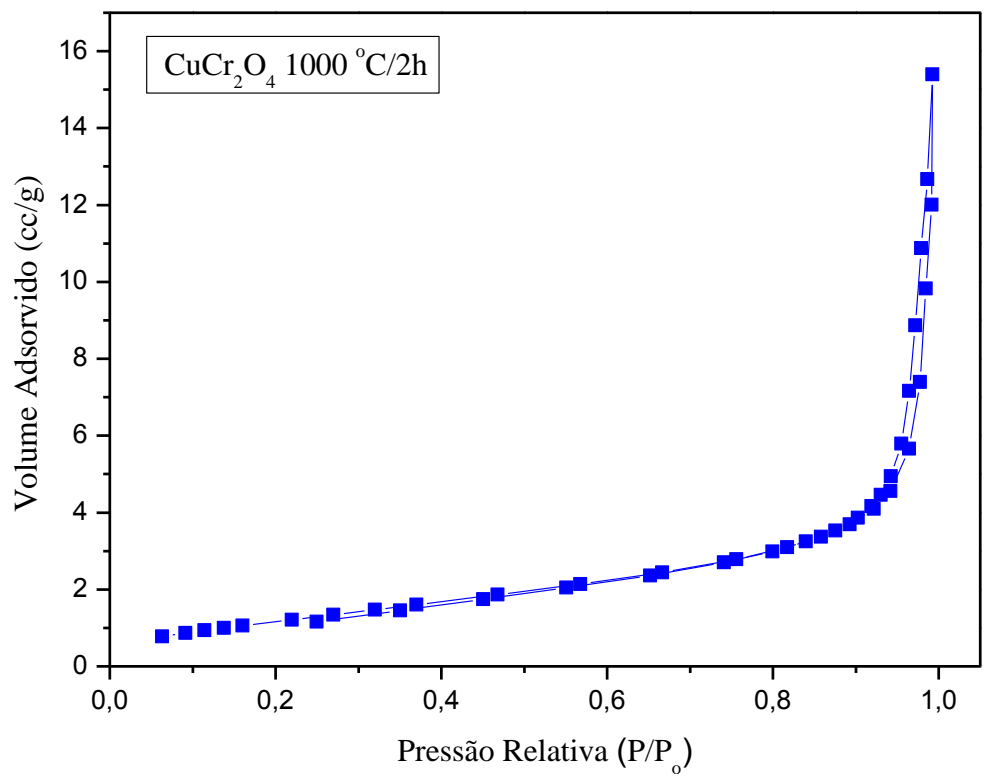

Figura 4. Isoterma de adsorção-dessorção do $\mathrm{CuCr}_{2} \mathrm{O}_{4}$ calcinado a $1000^{\circ} \mathrm{C}$ por 2 horas. 
Os resultados revelam ainda uma diminuição do diâmetro médio dos poros com o aumento da temperatura de tratamento, o que sugere a redução dos poros nas partículas e também uma tendência em sinterizar à baixa temperatura. A medida do diâmetro médio dos poros para o material calcinado a $1000{ }^{\circ} \mathrm{C}$ por 2 horas, exibida na Figura 5, indicam duas populações de poros, a primeira e menos pronunciada ocorre em torno de $80 \mathrm{~A}$ e a segunda e predominante a cerca de $1000 \mathrm{~A}$.

Pode-se observar também uma diminuição na área superficial com o aumento da temperatura de tratamento, Figura 6, ocasionada pelo crescimento das partículas, o que vem corroborar com os resultados de MEV.

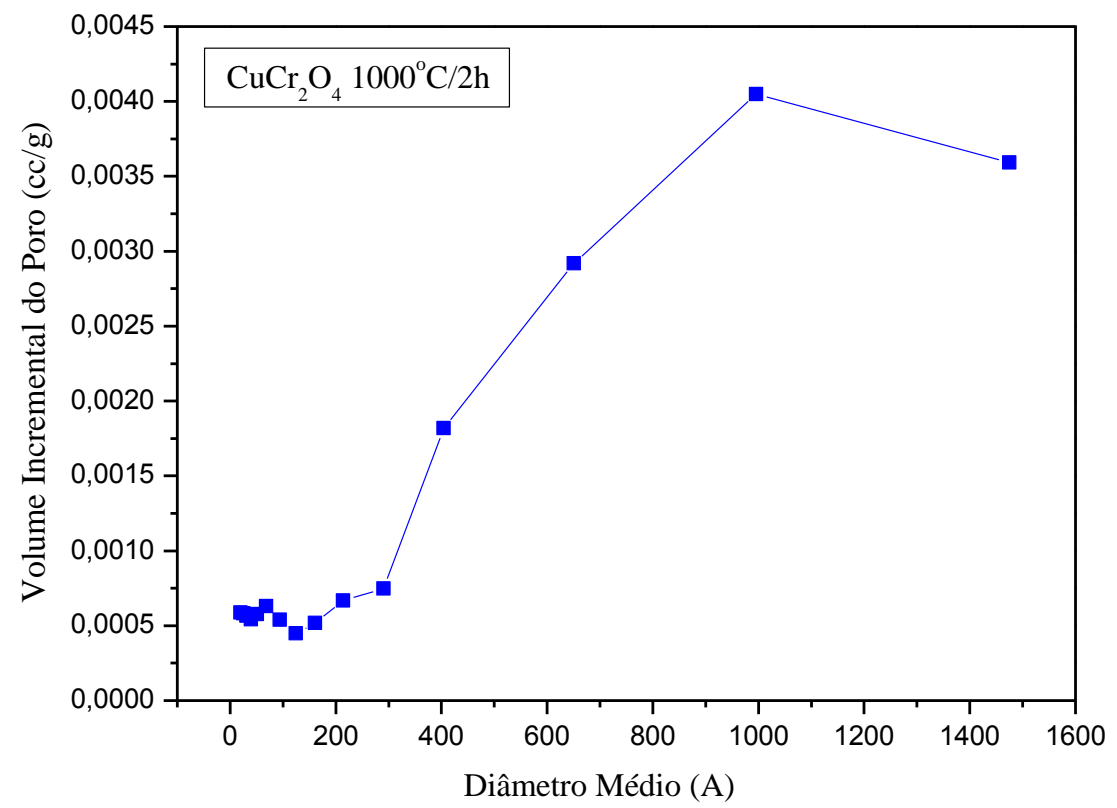

Figura 5. Distribuição de tamanho de poros do $\mathrm{CuCr}_{2} \mathrm{O}_{4}$ calcinado a $1000{ }^{\circ} \mathrm{C}$ por 2 horas.

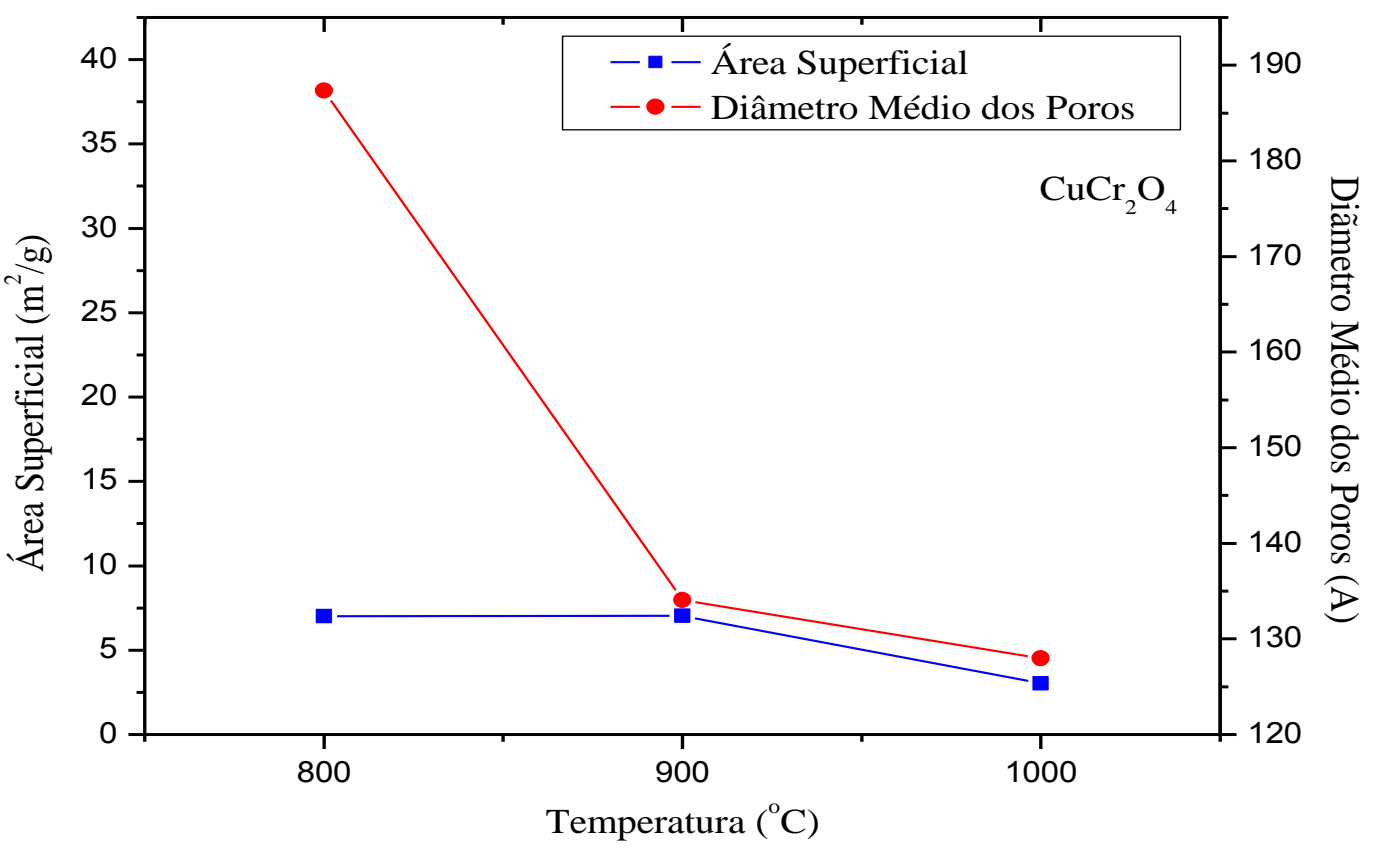

Figura 6. Diâmetro médio dos poros e área superficial em função da temperatura de calcinação do $\mathrm{CuCr}_{2} \mathrm{O}_{4}$. 


\section{Espetroscopia de absorção na região do UV-visível}

Os resultados das análises de refletância difusa na região do UV-visível das amostras, observadas na Figura 7, demonstram a presença de bandas de reflectância do $\mathrm{Cr}^{3+}$ em sítio octaédrico, bem definidas com maior intensidade próximo de $415 \mathrm{~nm}$, característico da transição ${ }^{4} \mathrm{~A}_{2 \mathrm{~g}}\left({ }^{4} \mathrm{~F}\right) \rightarrow{ }^{4} \mathrm{~T}_{1 \mathrm{~g}}\left({ }^{4} \mathrm{~F}\right)$, em $540 \mathrm{~nm}$ característico da transição ${ }^{4} \mathrm{~A}_{2 \mathrm{~g}}\left({ }^{4} \mathrm{~F}\right) \rightarrow{ }^{4} \mathrm{~T}_{2 \mathrm{~g}}\left({ }^{4} \mathrm{~F}\right)$, e menos pronunciada entre 660 e $730 \mathrm{~nm}$, atribuída as transições spin proibidas ${ }^{4} \mathrm{~A}_{2 g}\left({ }^{4} \mathrm{~F}\right) \rightarrow{ }^{4} \mathrm{~T}_{1 \mathrm{~g}}\left({ }^{2} \mathrm{G}\right)$ e ${ }^{4} \mathrm{~A}_{2 \mathrm{~g}}\left({ }^{4} \mathrm{~F}\right) \rightarrow{ }^{2} \mathrm{E}\left({ }^{2} \mathrm{G}\right)$. Pode-se verificar também a redução da reflectância na região do visível com o aumento na temperatura de calcinação do material, evidenciando a formação de pigmentos em tons escuros, sugerindo a predominância da fase espinélio.

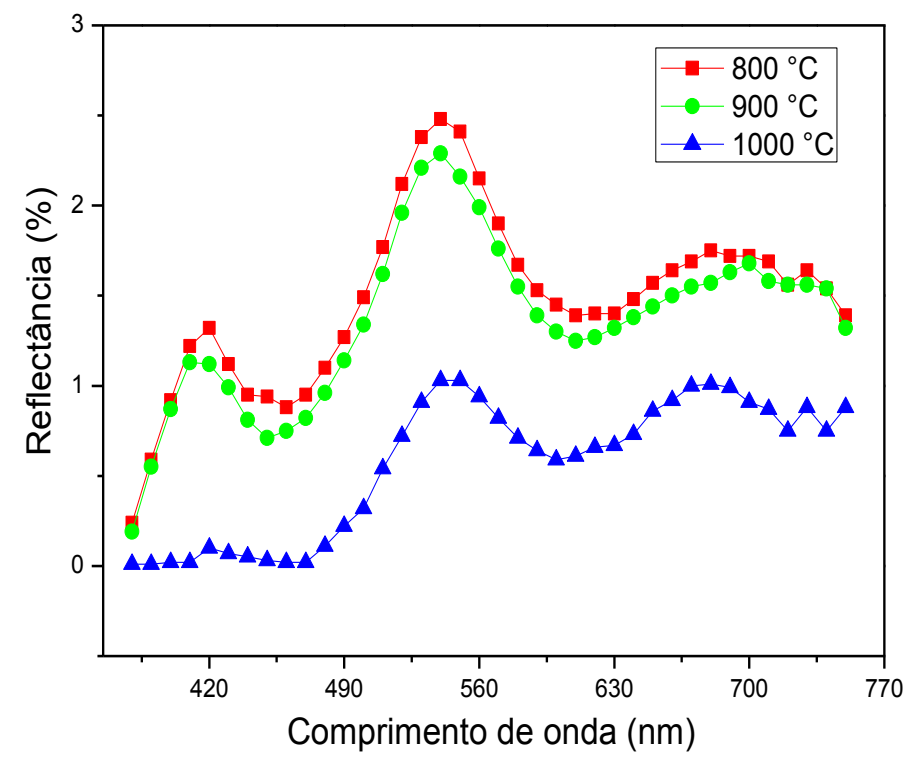

Figura 7. Espectro de reflectância difusa do pigmento obtido nas temperaturas de calcinação de $800^{\circ} \mathrm{C}$, $900^{\circ} \mathrm{C}$ e $1000^{\circ} \mathrm{C}$ por 2 horas.

Os resultados observados por meio das coordenadas colorimétricas, exibidas na Tabela 2, indicam a redução no valor de $\mathrm{L}$ com o aumento da temperatura de calcinação, o que sugerem a obtenção de pigmentos mais escuros. Para o parâmetro a* (que varia do verde ao vermelho) verifica-se redução da cor verde com a elevação da temperatura de calcinação e o parâmetro b* (que varia do azul ao amarelo) apresenta elevação da cor amarelo com o aumento na temperatura de calcinação do pigmento.

Tabela 2 - Coordenadas colorimétricas dos pós pigmentantes calcinados nas temperaturas de 800, 900 e $1000^{\circ} \mathrm{C}$ durante 2 horas.

\begin{tabular}{|c|c|c|c|c|}
\hline $\mathrm{T}\left({ }^{\circ} \mathrm{C}\right)$ & $\mathrm{L}^{*}$ & $\mathrm{a}^{*}$ & $\mathrm{~b}^{*}$ & $\Delta \mathrm{E}$ \\
\hline 800 & 14.487 & -5.085 & 8.510 & 17554,2 \\
\hline 900 & 13.607 & -5.035 & 9.210 & 17185,0 \\
\hline 1000 & 6.710 & -1.749 & 10.387 & 12488,9 \\
\hline
\end{tabular}


Os resultados da aplicação de $3 \%$ em massa do pigmento obtido e $97 \%$ em massa de frita e ligante orgânico e calcinados a temperatura de $1180^{\circ} \mathrm{C}$ durante 1 hora e aplicado em placa cerâmica, demonstrados nas Figuras 8 a 10, revelam estabilidade do pigmento obtido com o vidrado escolhido.

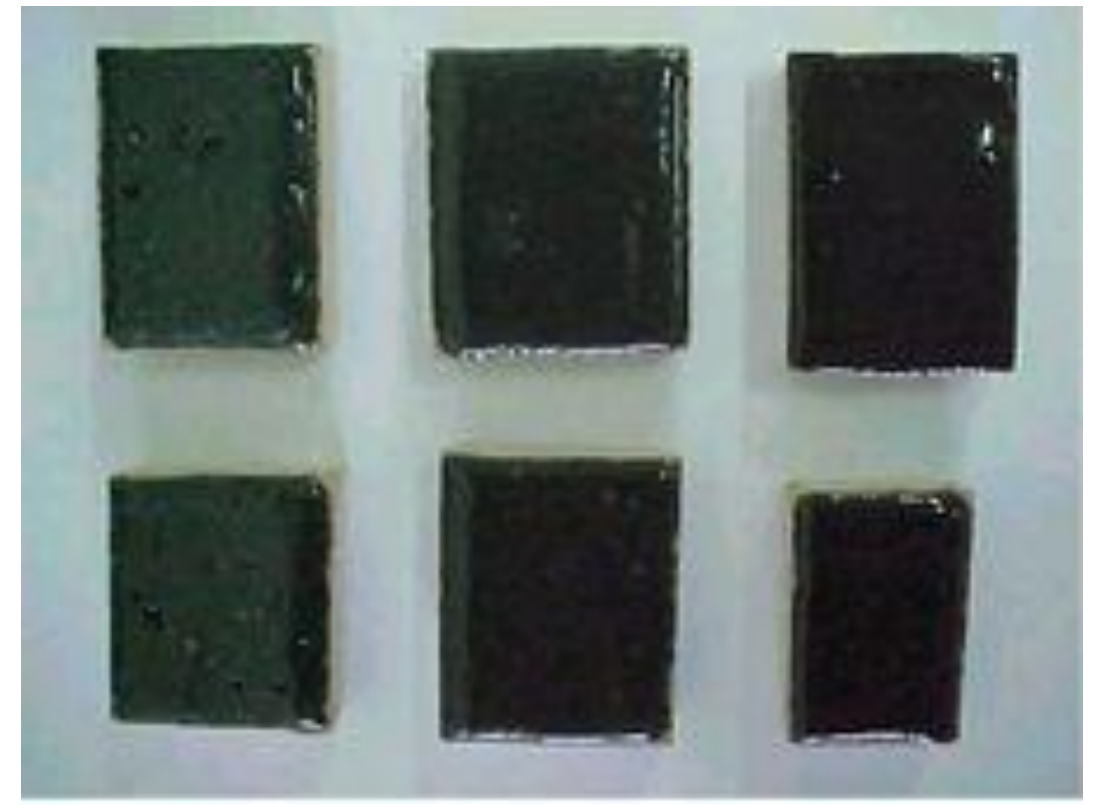

Figura 8. Aplicação, em peças cerâmicas, do pigmento obtido a 900 ㄷ.

\section{CONCLUSÃO}

Resinas precursoras se mostram adequadas para a preparação de pós pigmentantes homogêneos de Cromita de Cobre $\left(\mathrm{CuCr}_{2} \mathrm{O}_{4}\right)$, por se apresentarem estáveis com relação aos metais cromóforos presentes. Entretanto, é importante salientar o ocorrência de duas fases indexadas pelos picos de Eskolaita $\left(\mathrm{Cr}_{2} \mathrm{O}_{3}\right)$ e Cromita $\left(\mathrm{CuCr}_{2} \mathrm{O}_{4}\right)$ pela rota de preparação utilizada embora os diversos trabalhos observados na literatura sobre o tema descrevam a obtenção do $\mathrm{CuCr}_{2} \mathrm{O}_{4}$ por diferentes rotas de preparação (HARRISON, 2002; LIANG, 2005; DOLLASE, 1997) e sempre com a presença de mais de uma fase.

A análise morfológica realizada por meio da isoterma de adsorção/dessorção de N2 juntamente com o MEV indica que o aumento da temperatura de tratamento dos pós pigmentantes provoca aumento no tamanho das partículas e sugere a formação de agregados, contribuindo para a diminuição da área superficial. $O$ aumento da temperatura provoca também diminuição do diâmetro médio dos poros o que sugere à presença de poros nas partículas e uma tendência em sinterizar à baixa temperatura.

O pigmento obtido apresenta coloração verde, sendo este óxido um opacificante dentro de um sistema vítreo. Para todos os pigmentos obtidos pelo método Pechini houve uma baixa reflectância, evidenciando a formação de pigmentos em tons escuros. As Cores mais escuras foram formadas pelos materiais obtidos em temperaturas de 900 e 1000 ㄷ.

Pôde-se observar que os pós obtidos nas temperaturas de calcinação de 800,900 e $1000{ }^{\circ} \mathrm{C}$ não apresentaram alteração significativa na cor com a elevação da temperatura e que os resultados observados neste trabalho corroboram com os observados na literatura (ALMEIDA, 2007) para pigmentos a base de óxido de cobre. 


\section{REFERENCIAS}

1. ALMEIDA, R. N.; SANTOS, J. F.; SAMPAIO, J. A.; LUZ, A. B.; OGASAWARA, T.; ANDRADE, M. C.; Síntese de Pigmentos Cerâmicos por Precipitação Química. Cerâmica, 53 (2007) 57-61.

2. AMORÓS, J. L. Vidrados para Pavimentos e Revestimentos Cerâmicos: Evolução e Perspectivas. Cerâmica Industrial, 6 (6), novembro/dezembro 2001.

3. BONDIOLI, I.; MANFREDINI, T.; OLIVEIRA, A. P. N. de; Pigmentos Inorgânicos: projeto, produção e aplicação industrial. Cerâmica Industrial, 3 (4 -6) julho/dezembro, 1998.

4. CUI, H.; ZAYAT, M. and LEVY, D. Sol-Gel Synthesis of Nanoscaled Spinels Using Propylene Oxide as a Gelation Agent, Journal of Sol-Gel Science and Technology, 35 (2005), p. $175-181$.

5. DOLLASE, W. A.; O'NEILL, H. S. C.; The Spinels $\mathrm{CuCr}_{2} \mathrm{O}_{4}$ and $\mathrm{CuRh}_{2} \mathrm{O}_{4}$. Acta Crystallographica. (1997) C53, $657-659$.

6. DONDI, M.; CRUCIANI, G.; GUARINI, G.; MATTEUCCI, F. and RAIMONDO, M. The role of counterions ( $\mathrm{Mo}, \mathrm{Nb}, \mathrm{Sb}, \mathrm{W}$ ) in $\mathrm{Cr}-, \mathrm{Mn}-$, Ni- and $\mathrm{V}$-doped rutile ceramic pigments Part 2. Colour and technological properties, Ceramics International 32 (2006), p. 393405.

7. HARRISON, P. G.; ALLISON, F. J.; DANIELL, W.; Effect of Preparation Route and Thermal Treatment on the Nature of Copper and Chromium Doubly Promoted Ceria Catalysts. Chem. Mater. 2002, 14, 499 507.

8. LIANG, J.; PENG, Q.; WANG, X.; ZHENG, X.; WANG, R.; QIU, X.; NAN, C.; LI, Y.; Chromate Nanorods/Nanobelts: General Synthesis, Characterization and Properties. Inorganic Chemistry. 2005, $44,9405-9415$.

9. LIEC, Laboratório de Cerâmica. Disponível em: $<w w w$.liec.ufscar.br $>$ acesso em: 01.12.2003.

10. MELCHIADES, F. G.; BOSCHI, A. O. Cores e Tonalidades em Revestimentos cerâmicos. Cerâmica Industrial, $4(1-6)$ janeiro/dezembro, 1999.

11. OZEL, E.; SERVET, T. Production and Characterisation of Iron-Chromium Pigments. Journal of the European Ceramic Society, 23, 2097-2104, 2003.

12. PECHINI, M. Method of Preparing Lead and Alkaline - Earth Titanates and Niobates and Coating Method Using the Same to Form a Capacitor, U.S. Pat., no 3.330.697, 1967.

13. SILVA, R. F. L. Influência do contra-íon molibdênio nas propriedades do pigmento SnO2:Cr, 2008, João Pessoa, Dissertação (Mestrado em Química) - Universidade Federal da Paraíba, João Pessoa, 2008.

\section{AGRADECIMENTOS}

A FAPEMA pela concessão de bolsa, ao LIEC por viabilizar a realização das análises e a Professora Mackleia Mayara Oliveira da Silva pela revisão. 